\title{
CONCORDÂNCIA ENTRE OS FORMATOS IMPRESSO E ELETRÔNICO DO IPAQ-L
}

\author{
CONSISTENCY BETWEEN PRINT AND ELECTRONIC IPAQ-L FORMATS \\ COHERENCIA ENTRE LOS FORMATOS IMPRESO Y ELECTRÓNICO DEL IPAQ-L
}

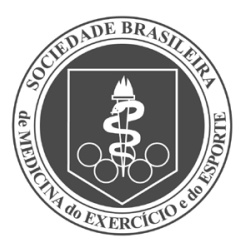

Artigo Original
Andréia Antonia Padilha Pires ${ }^{1}$ (Educador Físico)

Raymundo Pires Junior? (Educador Físico)

Rodrigo Franco de Oliveira' (Fisioterapeuta)

1. Educação Física da Universidade Norte do Paraná (Unopar), Londrina, PR, Brasil.

\section{Correspondência:}

Rua Milton Gavetti, 80, 86050-720, Londrina, PR, Brasil. padilinha36@gmail.com

\section{RESUMO}

Introdução: Existe uma diversidade de questionários para mensurar o nível de atividade física. O Questionário Internacional da Atividade Física (IPAQ) vem sendo amplamente utilizado, entretanto, o mesmo ainda não foi validado no formato eletrônico. Objetivo: Identificar a concordância entre o IPAQ versão longa no formato impresso e versão eletrônica. Métodos: Participaram do estudo 218 alunos de uma universidade privada, sendo 109 de cada gênero (idade $\leq 25$ e $\geq 26$ ). Na primeira etapa, os participantes receberam informações prévias sobre o preenchimento do formulário IPAQ-L (versão impressa), após esta etapa foi realizado o preenchimento da versão eletrônica (Weblpaq). Resultados: Nenhuma diferença significativa foi encontrada na comparação entre a versão impressa e eletrônica nas seções do IPAQ-L. O mesmo ocorreu quanto à Prática Habitual da Atividade Física (PHAF) para as proporções analisadas mediante qui-quadrado $\left(X^{2}\right)$. Reprodutibilidade excelente foi verificada através do coeficiente de correlação intraclasse (CCI), alcançando valores superiores a 0,82 (IC95\%: 0,68-0,90, idade $\geq 26$ anos) e 0,90 (IC95\%: 0,87-0,93) na análise conjunta. No índice Kappa ponderado a concordância foi substancial, ficando entre 0,62 (IC95\%: 0,41-0,77, gênero masculino) a 0,67 (IC95\%: 0,53-0,81, idade $\leq 25$ anos), demonstrando elevada concordância na análise de Bland e Altman. Conclusão: Diante dos resultados encontrados nesse estudo, pode-se inferir que o formato eletrônico do IPAQ-L atende aos pressupostos de reprodutibilidade e concordância, oferecendo alternativa moderna e eficaz nas análises da Prática Habitual da Atividade Física.

Palavras-chave: atividade física, questionários, estatística, avaliação.

\section{ABSTRACT}

Introduction: There is a variety of questionnaires to measure the level of physical activity. The International Physical Activity Questionnaire (IPAQ) has been widely used; however, this has not been validated in electronic format yet. Objective: To identify the correlation between the IPAQ long version in print and electronic versions. Methods: Study participants were 218 students at a private university, 109 of each gender (age $\leq 25$ and $\geq 26$ ). In the first stage, the participants were given prior information about filling out the form IPAQ-L (printed version); after that they completed the electronic version (Weblpaq). Results: No significant difference was found on comparing the print and electronic versions of the IPAQ-L. The same occurred in relation to the Practice of Habitual Physical Activity (PHAF) for the ratios by chi-square (X2). Excellent reproducibility was verified by the intraclass correlation coefficient (ICC), reaching values higher than 0.82 (95\% Cl: $0.68-0.90$, age $\geq 26$ years) and 0.90 (95\% Cl:0.87-0.93) in the combined analysis. In the weighted Kappa index agreement was substantial, ranging from 0.62 (95\% Cl: 0.41 to 0.77 , males) to 0.67 ( $95 \%$ Cl: 0.53 to 0.81 , age $\leq 25$ years). This shows high agreement on the Bland and Altman analysis. Conclusion: Considering the results of this study, it can be inferred that the electronic format of the IPAQ-L meets the assumptions of reproducibility and agreement, providing modern and effective alternative in the analyzes of Practice of Habitual Physical Activity.

Keywords: physical activity, questionnaires, statistics, assessment.

\section{RESUMEN}

Introducción: Hay una variedad de cuestionarios para medir el nivel de actividad fisica. El International Physical Activity Questionnaire (IPAQ) ha sido ampliamente utilizado, sin embargo, aún no ha sido validado en formato electrónico. Objetivo: Identificar la correlación entre la versión larga del IPAQ en versión impresa y versión electrónica. Métodos: Los participantes fueron 218 estudiantes de una universidad privada, 109 de cada sexo (edad $\leq 25$ y $\geq 26$ ). En la primera etapa, los participantes recibieron informaciones previas; antes de llenar el formulario de IPAQ-L (versión impresa) luego llenaron la versión electrónica (Weblpaq). Resultados: No se encontraron diferencias significativas al comparar las versiones impresa y electrónica del IPAQ-L. Lo mismo ocurrió en la práctica habitual de actividad física (PHAF) para las proporciones analizadas porchi-cuadrado $\left(X^{2}\right)$. Se encontró una excelente reproducibilidad mediante el coeficiente de correlación intraclase (CCI), alcanzando valores superiores a 0,82 (IC 95\%: 0,68-0,90, edad $\geq 26$ años) y 0,90 (IC 95\%: 0,87- 0,93) en el análisis combinado. La concordancia fue sustancial con el indice Kappa ponderado, pasando de 0,62 (IC 95\%: 0,41-0,77, varones) a 0,67 (IC 95\%: 0,53-0,81, edad $\leq 25$ años), lo que demuestra alto nivel de concordancia en el análisis de Bland y Altman. Conclusión: Teniendo en cuenta los resultados de este estudio, se puede inferir que el formato electrónico del IPAQ-L cumple con los supuestos de reproducibilidad y concordancia, proporcionando una alternativa moderna y eficaz en los análisis de la práctica habitual de actividad física.

Palabras clave: actividad física, cuestionarios, estadísticas, evaluación. 


\section{INTRODUÇÃO}

Atualmente o mundo vem sendo alertado sobre os riscos associados a níveis de atividade física insuficiente ${ }^{1,2}$. Informações sobre os benefícios da prática habitual de atividade física estão disseminadas nos mais diferentes grupos populacionais e em vários países. Evidências demonstram que manter-se ativo fisicamente aumenta a expectativa de vida independente, e acredita-se que mesmo um pequeno aumento do nível da atividade física pode representar uma melhoria na saúde ${ }^{3,5}$. Mesmo assim, os estudos mostram que níveis insuficientes de atividade física ocorrem em grandes proporções nas mais diferentes faixas etárias, tanto em países desenvolvidos como também no Brasil ${ }^{6,7}$.

Os levantamentos estatísticos são realizados mediante utilização de instrumentos que tem por finalidade avaliar o nível de atividade física, que podem ser: aqueles que utilizam informações fornecidas pelos próprios sujeitos (questionários, entrevistas e diários), os que utilizam de indicadores fisiológicos (consumo de oxigênio e frequência cardíaca) ou sensores de movimento que registram certas características das atividades realizadas num período de tempo ${ }^{8}$. Estes instrumentos, como também os marcadores de água duplamente marcada, o Computer Science Apliccation (CSA), são de alto custo para pesquisa epidemiológica, necessitando de cuidados e preocupações que poderiam influenciar no resultado, assim os questionários tem sido amplamente utilizados pela sua aplicabilidade para diferentes grupos, com baixo custo e por permitir coletar informações precisas sobre o tipo de atividade e o contexto em acontecem 8,10 .

Há uma grande diversidade de questionários para mensurar os níveis de atividade física ${ }^{11}$, o instrumento que vem sendo amplamente utilizado na área de atividade física é o Questionário Internacional de Atividade Física (International Physical Activity Questionnaire, IPAQ) 8, 12,13, composto por 27 questões no formato longo e oito questões no formato curto, proposto pela Organização Mundial da Saúde (OMS) em 1998 e testado no ano de 2000 em 12 países, entre eles o Brasil, sendo, portanto, validado e traduzido para a língua portuguesa ${ }^{14,15}$.

Sua aplicação consiste em preencher um formulário impresso em diferentes domínios como trabalho, tarefas domésticas, transporte, lazer, prática de exercício físico e esporte. Após seu preenchimento os MET's (Equivalente Metabólico = 1 MET: 3,5 ml/ $\mathrm{kg} / \mathrm{min}$ ) são calculados manualmente ou são tabulados em softwares que permitam tal procedimento para cada dimensão, bem como apresenta um escore total ${ }^{13,14}$.

Com o avanço tecnológico, a informática vem dominando todos os segmentos de nossas vidas ${ }^{16}$, por outro lado, boa parte das pesquisas ainda não utiliza os recursos da informática a seu favor. Faz-se necessários instrumentos que possam abranger de forma globalizada um maior número de pessoas e suas informações processadas (interpretadas) de maneira rápida, eficiente, com economia de tempo e financeira, indo ao encontro das questões ecológicas ${ }^{17}$, contribuindo para dinamizar o trabalho de pesquisadores na obtenção dos dados e resultados ${ }^{18}$. Possibilita envolvimento de diferentes grupos, diferentes localizações e maior número de participantes, permitindo ações mais eficazes mediante este instrumento de grande importância para a área epidemiológica e a sociedade, que sofre com as doenças da modernidade em decorrência de níveis insuficientes de atividade física9,10.

O objetivo deste estudo, portanto, foi identificar a concordância entre o questionário IPAQ versão longa no formato impresso (VI) e versão eletrônica (VE) denominado Weblpaq.

\section{MÉTODOS}

A amostra para esse estudo foi composta por estudantes de graduação das áreas de humanas e biológicas de uma instituição privada da cidade de Londrina, Paraná, Brasil, selecionados pelo método não probabilístico. Após serem informados do objetivo do estudo foram convidados a participar, e aqueles que aceitaram assinaram o Termo de Consentimento Livre e Esclarecido (TCLE). O projeto foi aprovado pelo Comitê de Ética Local, sob número 209.531.

Na primeira etapa, onde receberam as informações prévias sobre o preenchimento do formulário IPAQ-L (versão impressa), 374 estudantes responderam o instrumento. Na segunda etapa que consistiu no preenchimento da versão eletrônica (http://www.webipaq.com.br) em até dois dias após terem respondido a versão impressa, 218 concluíram os dois instrumentos, sendo 109 de cada gênero. A identificação dos instrumentos (versão impressa e eletrônica) do mesmo sujeito foi realizada mediante anotação do número de matrícula.

Avaliação da Atividade Física IPAQ-L Versão Impressa: O IPAQ é um instrumento que permite estimar o tempo semanal ou METs empregado na realização de atividades físicas em diferentes contextos da vida. Seu formato original é de preenchimento em papel e o cálculo para obtenção dos resultados é realizado manualmente ${ }^{12,14}$. Nas seções que compõem o instrumento é anotado o número de dias por semana e o tempo gasto em horas e minutos em determinada atividade física, na hipótese da não realização, deverá ser assinalada a alternativa "nenhum", subsequentemente os espaços de horas e minutos deverão ser deixados em branco. Posteriormente essas informações são utilizadas para cálculo em fórmulas específicas para cada seção de atividade física (trabalho, transporte, tarefas domésticas, lazer e esportes), com resultados expressos em METs em três níveis de atividade: caminhada, moderada e vigorosa. Finalmente esses resultados produzem um escore final classificando o respondente nos níveis de atividade física baixo, moderado e alto ${ }^{13}$.

IPAQ-L Versão Eletrônica: A versão eletrônica do questionário denominada Weblpaq foi desenvolvida por meio de um aplicativo da Internet (Web) utilizando linguagem de programação Hypertext Preprocessor 5 (PHP 5), script Java Script 20. O mesmo possui interface gráfica e linguagem PHP com eficiente suporte matemático, sistema multiplataforma, suporte a um grande número de banco de dados e código fonte aberto. As tecnologias de informação (TI) proporcionam automação das ferramentas de coletas de dados via internet de forma interativa ${ }^{19}$.

O Weblpaq é composto pela mesma estrutura do instrumento impresso, diferenciando apenas o modo de interface de preenchimento das respostas. Por exemplo, naquelas onde há alternativa "nenhum", o usuário é levado para a próxima questão sem precisar deixar em branco os espaços de horas e minutos. Os resultados são expressos em minutos, METs e nível de atividade física por seção: caminhada, moderada e vigorosa, assim como oferece escore final do inquérito classificando igualmente como a versão impressa.

\section{Análise estatística}

O tratamento estatístico dos dados foi realizado mediante pacote computadorizado Statistical Package for the Social Science (SPSS), versão 20.0. Inicialmente, para caracterização da amostra quanto à prática habitual de atividade física utilizou-se de recursos da estatística não-paramétrica, uma vez que os dados apresentados não mostraram distribuição de frequência normal mediante utilização do teste de Kolmogorov-Smirnov. Quanto à quantidade de METs equivalente às quatro dimensões da prática habitual de atividade física (trabalho, transporte, tarefas domésticas e lazer/exercício físico) recorreu-se aos procedimentos da estatística descritiva (mediana e diferença interquartil) e, posteriormente, para estabelecer comparações entre sexo e idade, à análise de variância por postos de Kruskal-Wallis (K-W). O nível de significância foi fixado em 5\% ( $p<0,05)$. As proporções de 
universitários reunidos nas três categorias de prática habitual de atividade física propostas pelo IPAQ (baixa, moderada e elevada) foram analisadas mediante tabelas de contingências, envolvendo teste de qui-quadrado $\left(X^{2}\right)$ para identificação das diferenças estatísticas entre sexo e idade. Na sequência, para estabelecer a concordância entre as respostas dos universitários apresentadas mediante os formatos impresso e eletrônico do IPAQ, foi utilizado o índice Kappa ponderado e o coeficiente de correlação intraclasse (ICC), acompanhado da plotagem do diagrama de dispersão de Bland-Altman²0.

\section{RESULTADOS}

Os resultados descritivos são apresentados na tabela 1 por meio da mediana e intervalo interquartil (Q3-Q1) para os grupos etários e gênero em METs semanais para as diferentes seções do IPAQ-L. A versão impressa do instrumento apresentou valores de METs mais elevados nas seções trabalho e transporte, enquanto que no formato eletrônico foram as seções tarefas domésticas e lazer/esportes que apresentaram os maiores escores. Na comparação das versões impressa e eletrônica realizada pela análise de variância por postos de Kruskal-Wallis (K-W), o resultado para idades até 25 anos foi de $p=0,91$ e nas idades acima de 26 anos $p=0,87$, enquanto que nos rapazes $p=0,99$ e para as moças $p=0,95$. Em nenhuma das seções para gênero e idade foi verificada diferença estatística para as versões impressa e eletrônica do IPAQ-L.

Na análise das proporções quanto à classificação dos níveis de atividade física (alto, moderado e baixo) para a versão impressa e eletrônica através do qui-quadrado (tabela 2), não ocorreu diferença significativa, uma vez que os resultados demonstraram significância de $p=0,45$ para o gênero feminino e de $p=0,90$ no masculino. Para aqueles com até 25 anos de idade $\mathrm{o}$ resultado foi de $p=0,16$ e para idades acima de 26 anos $p=0,84$.

Tabela 1. Valores medianos e diferenças interquartis (Q3-Q1) quanto aos METs despendidos em diferentes dimensões da atividade física nas versões impressa e eletrônica do IPAQ- $L$ ( $n=218)$.

\begin{tabular}{|c|c|c|c|c|c|c|c|c|}
\hline \multirow[b]{2}{*}{$\begin{array}{c}\text { Trabalho } \\
\text { Md (Q3-Q1) }\end{array}$} & \multicolumn{2}{|c|}{$\begin{array}{c}\text { Masculino } \\
\text { Impres. eletrôn. } \\
(n=109)(n=109)\end{array}$} & \multicolumn{2}{|c|}{$\begin{array}{c}\text { Feminino } \\
\text { Impres. eletrôn. } \\
(n=109)(n=109)\end{array}$} & \multicolumn{2}{|c|}{$\begin{array}{c}\leq 25 \text { anos } \\
\text { Impres. eletrôn. } \\
(n=163)(n=163)\end{array}$} & \multicolumn{2}{|c|}{$\begin{array}{c}\geq 26 \text { anos } \\
\text { Impres. eletrôn. } \\
(n=55)(n=55)\end{array}$} \\
\hline & $\begin{array}{c}4752 \\
(13074)\end{array}$ & $\begin{array}{c}3809 \\
(11352)\end{array}$ & $\begin{array}{c}1194 \\
(9120)\end{array}$ & $\begin{array}{c}805 \\
(9030)\end{array}$ & $\begin{array}{c}3276 \\
(11812)\end{array}$ & $\begin{array}{l}2410 \\
(9516)\end{array}$ & $\begin{array}{c}2034 \\
(10224)\end{array}$ & $\begin{array}{c}1211 \\
(10980)\end{array}$ \\
\hline $\begin{array}{c}\text { Transporte } \\
\text { Md (Q3-Q1) }\end{array}$ & $\begin{array}{c}297 \\
(1053)\end{array}$ & $\begin{array}{c}231 \\
(936)\end{array}$ & $\begin{array}{c}396 \\
(1080)\end{array}$ & $\begin{array}{l}396 \\
(990)\end{array}$ & $\begin{array}{c}423 \\
(1163)\end{array}$ & $\begin{array}{c}405 \\
(1212)\end{array}$ & $\begin{array}{c}63 \\
(660)\end{array}$ & $\begin{array}{c}00 \\
(495)\end{array}$ \\
\hline $\begin{array}{c}\text { Tarefas D. } \\
\text { Md (Q3-Q1) }\end{array}$ & $\begin{array}{c}240 \\
(1800)\end{array}$ & $\begin{array}{c}240 \\
(1440)\end{array}$ & $\begin{array}{c}1440 \\
(3540)\end{array}$ & $\begin{array}{c}1560 \\
(3360)\end{array}$ & $\begin{array}{c}680 \\
(2730)\end{array}$ & $\begin{array}{c}720 \\
(2860)\end{array}$ & $\begin{array}{c}1120 \\
(3120)\end{array}$ & $\begin{array}{c}1200 \\
(3390)\end{array}$ \\
\hline $\begin{array}{c}\text { Lazer/esp. } \\
\text { Md (Q3-Q1) }\end{array}$ & $\begin{array}{c}1318 \\
(2022)\end{array}$ & $\begin{array}{c}1398 \\
(2040)\end{array}$ & $\begin{array}{c}219 \\
(1200)\end{array}$ & $\begin{array}{c}240 \\
(1170)\end{array}$ & $\begin{array}{c}720 \\
(2211)\end{array}$ & $\begin{array}{c}839 \\
(1866)\end{array}$ & $\begin{array}{c}640 \\
(2139)\end{array}$ & $\begin{array}{c}720 \\
(1930)\end{array}$ \\
\hline
\end{tabular}

Tabela 2. Proporção de prática habitual de atividade física proposta pelo IPAQ-L versão impressa e eletrônica para gênero e idade ( $n=218)$.

\begin{tabular}{|c|c|c|c|c|c|c|c|c|}
\hline & \multicolumn{2}{|c|}{$\begin{array}{c}\text { Masculino } \\
(n=109)\end{array}$} & \multicolumn{2}{|c|}{$\begin{array}{c}\text { Feminino } \\
(n=109)\end{array}$} & \multicolumn{2}{|c|}{$\begin{array}{c}\leq 25 \text { anos } \\
(n=163)\end{array}$} & \multicolumn{2}{|c|}{$\begin{array}{c}\geq 26 \text { anos } \\
(n=55)\end{array}$} \\
\hline & \multicolumn{2}{|c|}{$\begin{array}{l}\text { Impresso } \\
\text { eletrônico } \\
\text { n (\%) n (\%) }\end{array}$} & \multicolumn{2}{|c|}{$\begin{array}{l}\text { Impresso } \\
\text { eletrônico } \\
\text { n (\%) n (\%) }\end{array}$} & \multicolumn{2}{|c|}{$\begin{array}{l}\text { Impresso } \\
\text { eletrônico } \\
\text { n (\%) n (\%) }\end{array}$} & \multicolumn{2}{|c|}{$\begin{array}{l}\text { Impresso } \\
\text { eletrônico } \\
\text { n (\%) n (\%) }\end{array}$} \\
\hline Alto & $\begin{array}{c}88 \\
(80,7)\end{array}$ & $\begin{array}{c}87 \\
(79,8)\end{array}$ & $\begin{array}{c}83 \\
(76,1)\end{array}$ & $\begin{array}{c}80 \\
(73,4)\end{array}$ & $\begin{array}{c}134 \\
(82,2)\end{array}$ & $\begin{array}{c}129 \\
(79,1)\end{array}$ & $\begin{array}{c}37 \\
(67,3)\end{array}$ & $\begin{array}{c}38 \\
(69,1)\end{array}$ \\
\hline Mod. & $\begin{array}{c}11 \\
(10,1)\end{array}$ & $\begin{array}{c}13 \\
(11,9)\end{array}$ & $\begin{array}{c}14 \\
(12,8)\end{array}$ & $\begin{array}{c}19 \\
(17,4)\end{array}$ & $\begin{array}{c}16 \\
(9,8)\end{array}$ & $\begin{array}{c}26 \\
(16,0)\end{array}$ & $\begin{array}{c}9 \\
(16,4)\end{array}$ & $\begin{array}{c}7 \\
(12,7)\end{array}$ \\
\hline Baixo & $\begin{array}{c}10 \\
(9,2)\end{array}$ & $\begin{array}{c}9 \\
(8,3)\end{array}$ & $\begin{array}{c}12 \\
(11,0)\end{array}$ & $\begin{array}{c}10 \\
(9,2)\end{array}$ & $\begin{array}{c}13 \\
(8,0)\end{array}$ & $\begin{array}{c}8 \\
(4,9)\end{array}$ & $\begin{array}{c}9 \\
(16,4)\end{array}$ & $\begin{array}{c}10 \\
(18,2)\end{array}$ \\
\hline
\end{tabular}

* $p<0,05$
A concordância nas respostas obtidas entre os universitários nos formatos impresso e eletrônico do IPAQ-L mediante o coeficiente de correlação intraclasse (CCl para os dados numéricos e o índice Kappa ponderado para os dados categóricos, e seus respectivos intervalos de confiança, são apresentados na tabela 3.

Tabela 3. Concordância entre as respostas dos universitários apresentadas mediante os formatos impresso e eletrônico do IPAQ.

\begin{tabular}{c|c|c|c|c}
\hline Variável & CCI & IC 95\% & Kappa & IC 95\% \\
\hline Todas & 0,90 & $0,87-0,93$ & 0,71 & $0,57-079$ \\
\hline Gênero & & & & \\
\hline Masculino & 0,94 & $0,91-0,96$ & 0,62 & $0,41-0,77$ \\
\hline Feminino & 0,87 & $0,81-0,91$ & 0,66 & $0,49-079$ \\
\hline Idades & & & & \\
\hline$\leq 25$ anos & 0,94 & $0,91-0,95$ & 0,67 & $0,53-0,81$ \\
\hline$\geq 26$ anos & 0,82 & $0,68-0,90$ & 0,64 & $0,41-0,81$ \\
\hline${ }^{*}, 0,05$.
\end{tabular}

Os resultados do CCl apresentaram valores de Alpha de Cronbach superiores a 0,82 (IC95\%: 0,68-0,90, para idade $\geq 26$ anos), chegando a 0,94 (gênero masculino IC95\%: 0,91-0,96 e idade $\leq 25$ anos IC95\%: 0,910,95) e 0,90 (IC95\%: 0,87-0,93) na análise conjunta entre as variáveis, o que indica reprodutibilidade excelente ${ }^{21}$ entre as respostas da versão impressa e eletrônica. Os resultados da estatística F alcançaram valores entre 5,42 a 17,03 e significância p<0,00 em todas as variáveis analisadas.

Para as variáveis categóricas analisadas mediante Kappa ponderado, os resultados estabelecidos ficaram entre 0,62 (IC95\%: 0,41-0,77 para o gênero masculino) a 0,67 (IC95\%: 0,53-0,81 para idade $\leq 25$ anos), alcançando concordância substancial22. Quando a análise envolveu todas as variáveis (gênero e idade) o valor de Kappa ponderado foi de 0,71 (IC95\%: 0,57-0,79), atingindo o mesmo nível de concordância.

Para determinar a concordância absoluta entre a versão impressa e eletrônica do IPAQ-L quanto à prática habitual da atividade física (PHAF) baixa, moderada e alta, o diagrama de dispersão de Bland e Altman é apresentado na figura 1 para o gênero masculino e feminino e na figura 2 para as idades $\leq 25$ anos e $\geq 26$ anos. Observou-se elevada concordância nos diagramas, uma vez que os valores médios encontrados tanto para os gêneros como para as idades nas versões impressa e eletrônica do IPAQ-L sempre estiveram próximos a zero e com reduzidos casos de dispersão além dos limites superior e inferior do desvio padrão das médias.

Entre os universitários do sexo masculino, a PHAF moderada parece ser aquela de maior concordância, apresentando diferença média entre as versões testadas de -65,7 (IC95\%: 1343,1 -1211,7) e limites de concordância variando de 13122,1 (+2DP) a -13253,5 (-2DP). Entre as mulheres, o mesmo raciocínio pode ser admitido para a PHAF baixa, com média de -144,2 (IC95\%: 386,3 -674,8) com limite superior de 5307,1 e inferior de $-5595,5$.

Nas idades $\leq 25$ anos os resultados foram similares, destacando-se a PHAF alta com apenas 5 casos que ultrapassaram os limites superior (+DP 6324,6) e inferior (-DP -6047,8), com média de 138,4 (IC95\%: 626,6 -349,8). Para as idades $\geq 26$ anos a dispersão nas três categorias de PHAF demonstrou ser mais elevada do que seus pares mais jovens.

\section{DISCUSSÃO}

Dentre os inúmeros questionários disponíveis para avaliar a atividade física, o IPAQ-L se destaca pela sua ampla utilização e propriedades psicométricas ${ }^{11}$. Seu preenchimento exige o cálculo das respostas de forma manual, tanto para o tempo despendido em atividades diária 


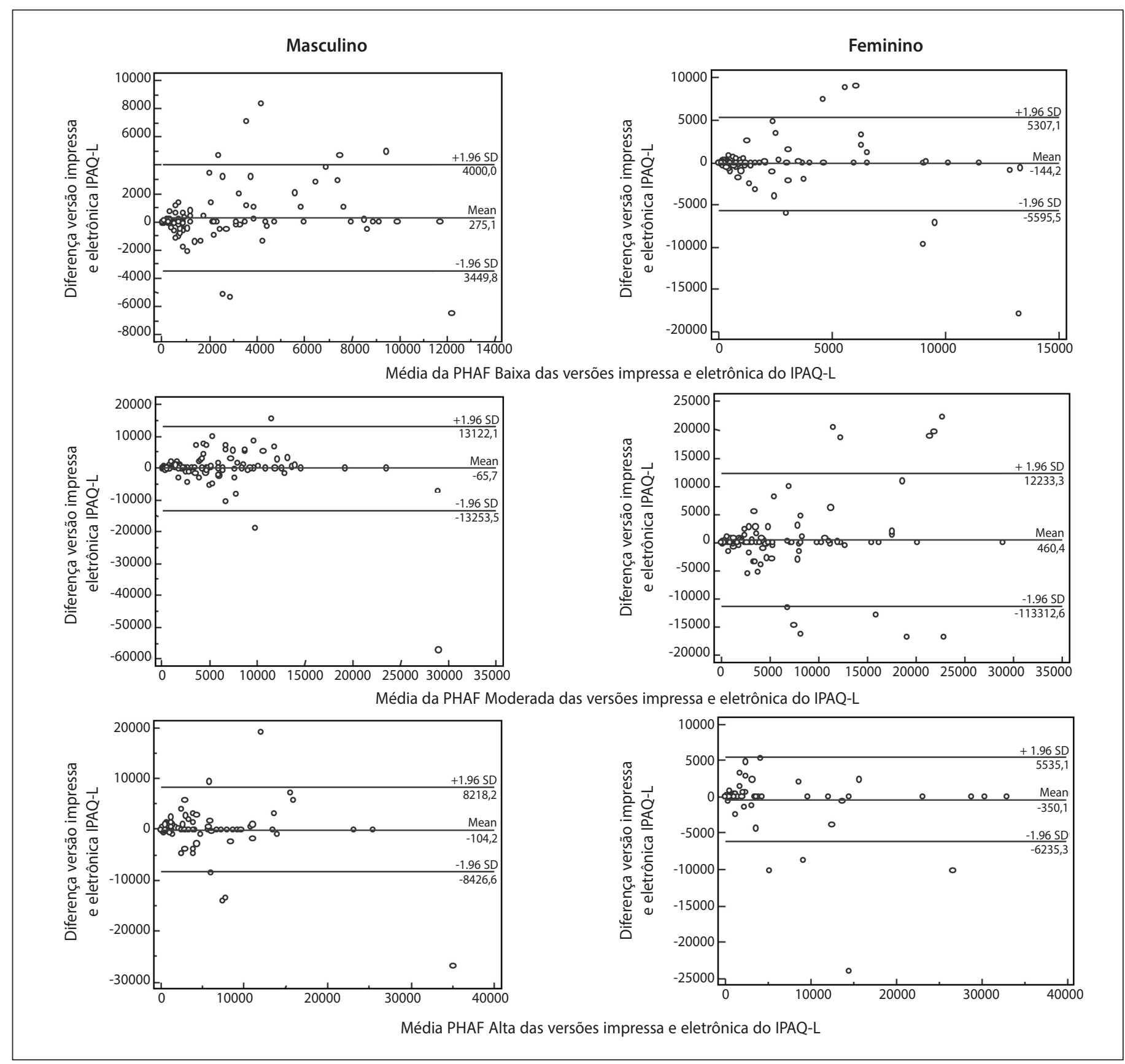

Figura 1. Diagrama de Bland e Altman quanto aos limites de concordância para o gênero masculino e femino entre a versão impressa e eletrônica do IPAQ-L.

ou semanal, como para classificar quanto ao nível de atividade física. Recursos tecnológicos mediante utilização da Internet podem oferecer maior velocidade na obtenção dos resultados, assim, este trabalho teve como finalidade identificar a concordância entre o IPAQ versão longa no formato impresso e eletrônico.

Nenhuma diferença estatística foi verificada nas variáveis numérica entre o formato impresso e eletrônico do IPAQ- $L$, demonstrando que a quantidade de METs ou minutos despendidos nas atividades em cada seção respondidos nos dois formulários foram coincidentes entre os grupos testados. O mesmo ocorreu quando os resultados foram expressos em categorias, uma vez que a classificação do nível de atividade física nos dois formatos do IPAQ-L foi semelhante entre os universitários, independente do gênero ou idade.

Na análise de concordância entre os questionários impresso e eletrônico, os valores de CCl apresentaram diferença estatística $(p<0,05)$ tanto para idade como gênero, alcançando reprodutibilidade excelente $(C C l=0,82$ a 0,94), apontando valores adequados para aplicação do
IPAQ-L versão eletrônica para adultos de ambos os gêneros. Em inquérito específico para avaliar a atividade física em crianças, a utilização da Internet também se mostrou eficiente com valores de $\mathrm{CCl}>0,84$ para todos os itens do instrumento utilizado ${ }^{23}$. Resultados favoráveis também foram identificados para utilização de questionário eletrônico com foco à saúde em adolescentes ${ }^{17,24}$ demonstrando ainda vantagem na segurança e confiança no inquérito via internet para respostas sigilosas para comportamentos de risco assumidos para esta população ${ }^{25}$. Quanto à proporção de sujeitos classificados nos diferentes níveis de atividade física através do IPAQ-L nos formatos papel e Internet, a concordância substancial mediante Kappa ponderado demonstrou grande similaridade para os dados agrupados K=0,71 (IC95\%: 0,57-0,79). Menor concordância observada para o gênero masculino $(K=0,62)$ no presente estudo, também foi observado em inquérito específico para avaliar atividade física entre adolescentes nos formulários papel x eletrônico. Enquanto os rapazes obtiveram concordância de $K=0,39$, as moças alcançaram $\mathrm{K}=0,51^{26}$. 


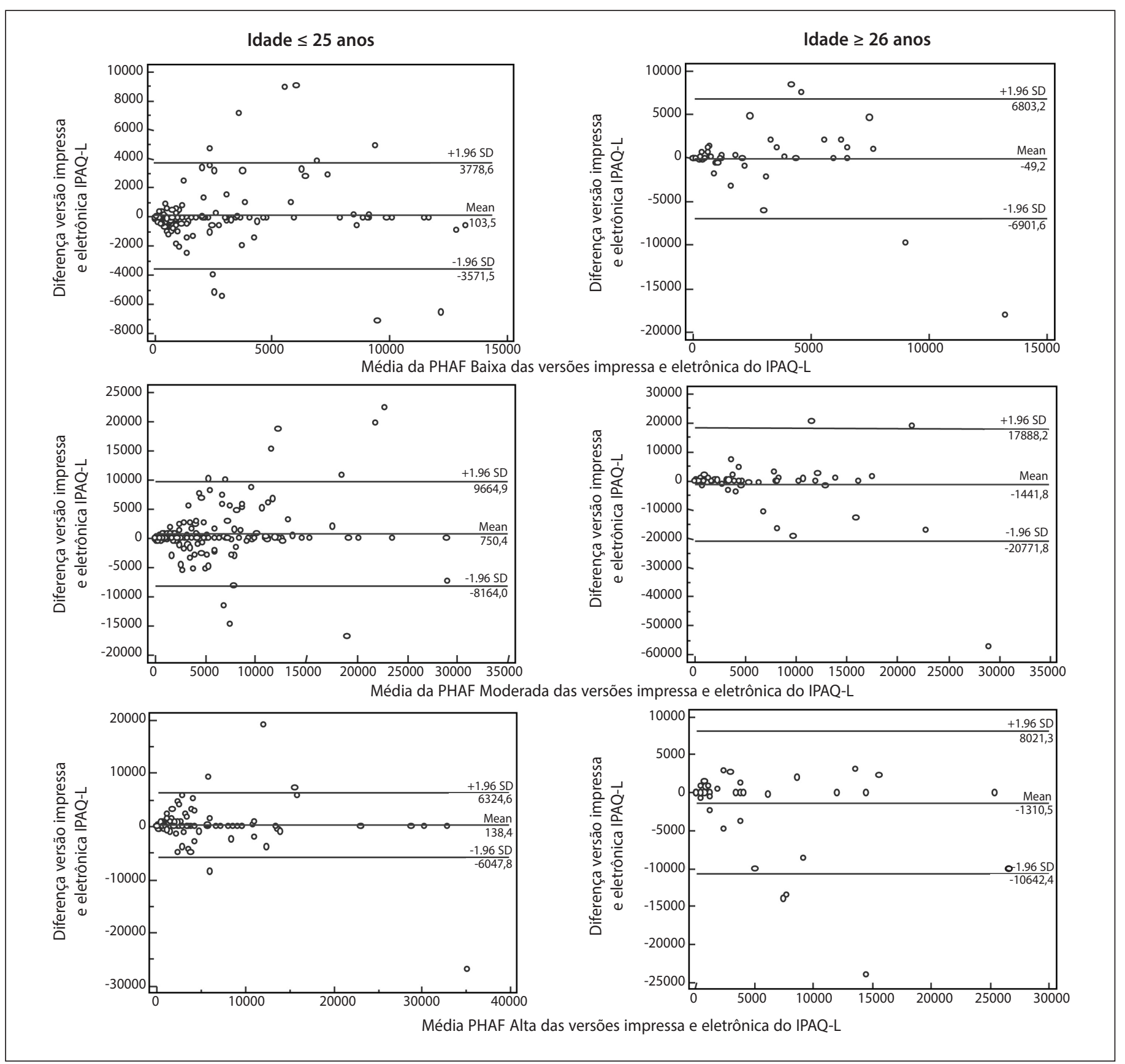

Figura 2. Diagrama de Bland e Altman quanto aos limites de concordância para as idades $\leq 25$ anos e $\geq 26$ anos entre a versão impressa e eletrônica do IPAQ-L.

Através do diagrama de dispersão de Bland e Altmann ${ }^{20}$, pode ser verificado com maior sensibilidade as diferenças entre as réplicas (papel versus Web). As figuras demonstraram para a idade e gênero que a média das diferenças sempre esteve próximo ao zero (-144,2 a 275,1 METs para o nível baixo, -1441,8 a 750,4 para o moderado e -13310,5 a 138,4 para o alto) e os valores das diferenças individuais ficaram na grande maioria dentro dos limites extremos do intervalo de confiança, indicando assim elevada concordância entre os instrumentos testados. Nota-se, porém, que as mulheres apresentaram dispersão pouco maior que os homens principalmente no nível moderado de atividade física, assim como os sujeitos com idade $\leq 25$ anos nos níveis baixo e moderado de prática habitual de atividade física. Mesmo assim, não sendo observado viés sistemático, com poucas diferenças individuais notadas. Em estudo envolvendo a concordância entre inquérito de atividade física no formato impresso versus eletrônico e acurácia por acelerômetro nos diferentes domínios (seções) da atividade física, foram estabelecidos grandes limites de concordância, mesmo que uma das seções apresentou várias diferenças individuais, porém, com baixo viés sistemático ${ }^{27}$.

Estudos desta natureza foram desenvolvidos para utilização de diferentes questionários no formato eletrônico principalmente para crianças e adolescentes ${ }^{17,23-26}$. Em adultos poucos estudos são encontrados na literatura que procuram estabelecer a validade de instrumentos via Internet ${ }^{27,28}$. As ferramentas eletrônicas podem favorecer a velocidade e precisão dos resultados, principalmente no campo epidemiológico, além da confiabilidade na tabulação e armazenamento dos dados. Quanto ao aspecto ambiental, reduz a utilização de papel e pode reduzir gastos. Deve ser considerado ainda que os erros de respostas pode acontecer com maior frequência quando um mesmo inquérito é utilizado no formato impresso (papel-caneta/lápis) comparado a um modelo eletrônico, podendo ser mais vantajoso ainda quando o questionário exigir o preenchimento de espaços em branco. A plataforma eletrônica impede que se avance para a próxima questão sem responder a anterior ${ }^{29}$. Permite também maior privacidade 
e anonimato, proporcionando maior honestidade e sensibilidade nas respostas, evitando, assim, viés nos resultados.

\section{CONCLUSÃO}

Mediante os resultados deste estudo, o IPAQ-L no formato eletrônico apresentou valores de concordância adequados tanto nas análises mais conservadoras, quanto nas mais sensíveis, permitindo sua aplicação em adultos de ambos os gêneros.

\section{AGRADECIMENTOS}

Os autores agradecem aos Professores Dartagnan Pinto Guedes (Unopar) e Enrique Garcia Bengoechea (McGill University) pela contribuição indispensável na construção deste trabalho.

Todos os autores declararam não haver qualquer potencial conflito de interesses referente a este artigo.

\section{REFERÊNCIAS}

1. Tenório MM, Barros MG, Tassitano RM, Bezerra J, Hallal PC. Atividade física e comportamento sedentário em adolescentes estudantes do ensino médio. Rev Bras Epidemiol. 2010;13(1):105-17.

2. Farias Junior JC. Prevalência e fatores de influência para inatividade física em adolescentes. Rev Bras Cien Mov. 2006;14(1):63-70.

3. Zanchetta LM, Barros, MA, César CG, Carandina L, Goldbaum M, Alves MP. Inatividade física e fatores associados em adultos, São Paulo, Brasil. Rev Bras Epidemiol. 2010;13(3):1-13.

4. Haskell WL, Lee IM, Pate RR, Powell KE, Blair SN, Franklin BA, et al. Physical Activity and Public Health: Updated Recommendation for Adults from the American College of Sports Medicine and the American Heart Association. Med Sci Sports Exerc. 2007;116:1081-93.

5. Gomes KV, Zazá DC. Motivos de Adesão a Prática de Atividade Física em Idosas. Rev Bras Ativ Fís Saúde. 2009;14(2):132-8.

6. Varo JJ, Martínez-Gonzalez MA, Irala-Estévez J, Kearney J, Gibney M, Martinez JA. Distribution and determinants of sedentary lifestyles in the European Union. Int J Epidemiol. 2003;32(1):138-46.

7. Masson CR, Dias-da-Costa JS, Olinto MA, Meneghel S, Costa CC, Bairros F, et al. Prevalência de sedentarismo nas mulheres adultas da cidade de São Leopoldo, Rio Grande do Sul, Brasil. Cad Saude Publica. 2005; 21(6):1685-94

8. Pardini R, Matsudo S, Araújo T, Matsudo V, Andrade E, Braggion G, et al. Validação do questionário internacional de nível de atividade física (IPAQ - versão 6): estudo piloto em adultos jovens brasileiros. Rev Bras Cien Mov. 2001;9(3):45-51

9. Barros MG, Nahas MG. Reprodutibilidade (teste-reteste) do questionário internacional de atividade física (QIAF-Versäo 6): um esudo piloto com adultos no Brasil. Rev Bras Cien Mov. 2000;8(1):23-6.

10. Farias Júnior JC, Siqueira FV, Nahas MV, Barros MG. Prevalência e fatores associados a níveis insuficientes de atividade física em jovens estudantes de duas cidades brasileiras: últimos sete dias e semana típica ou normal. Rev Bras Educ Fís Esporte. 2011;25(4):619-29.

11. Poppel MM, Chinapaw, MM, Mokkink LB, Mechelen W, Terwee CB. Physical Activity Questionnaires for Adults A Systematic Review of Measurement Properties. Sports Med. 2010;40(7):565-600

12. Pardini R, Matsudo SM, Matsudo VR, Araujo TA, Braggion GF, Andrade GR, et al. Validation of international physical activity questionnaire (IPAQ): pilot study in brazilian young adults. Med Sci Sports Exerc. 1997;29(06):5-9.

13. International Physical Activity Questionnaire (IPAQ). Processing and Analysis of the International Physical Activity Questionnaire (IPAQ) - Short and Long Forms. Disponivel em: http://www.ipaq. ki.se/scoring.pdf.

14. Matsudo S, Timóteo A, Victor M, Andrade D, Andrade E, Oliveira LC, et al. Questinário internacional de atividade f1sica(IPAQ): estudo de validade e reprodutibilidade no Brasil / International physical activity questionnaire (IPAQ): study of validity and reability in Brazil Rev Bras Ativ Fís Saúde. 2001;6(2):5-18.
15. Benedetti TB, Antunes PC, Rodriques-Añez CR, Mazo GZ, Petrosk EL. Reprodutibilidade e validade do Questionário Internacional de Atividade Física (IPAQ) em homens idosos. Rev Bras Med Esporte. 2007;1 (13):11-6.

16. Vasconcellos L, Guedes LA. E-Surveys: Vantagens e Limitações dos Questionários Eletrônicos via Internet no Contexto da Pesquisa Científica. In: Anais do Encontro Nacional de Pós-Graduação e Pesquisa em Administração. Rio de Janeiro. 2007;1-16.

17. Raat H, Mangunkusumo RT, Landgraf JM, Kloek G, Brug J. Feasibility, reliability, and validity of adolescent health status measurement by the Child Health Questionnaire Child Form (CHQ-CF): internet administration compared with the standard paper version. Qual Life Res. 2007;16:675-85.

18. Mangunkusumo RT, Duisterhout JS, Graaff N, Maarsingh EJ, Koning HJ, Raat H. Internet Versus Paper Mode of Health and Health Behavior Questionnaires in Elementary Schools: Asthma and Fruit as Examples. J Sch Health. 2006;76:80.

19. Samara BS, Barros JC. Pesquisa de marketing: conceitos e metodologia. São Paulo: Makron Books; 1997.

20. Bland JM, Altman DG. Measuring agreement in method comparison studies. Stat Methods Med Res 1999;8(2):135-60.

21. Fleiss JL. Reliability of measurement. In: The desing and analysis of clinical experiments. New York: John Wiley \& Sons; 1986

22. Landis JR, Koch GG. The measurement of observer agreement for categorical data. Biometrics. 1977;33:159-74.

23. Legnani $E$, Legnani RS, rech CR, Barros MG, Campos W, Assis MA. Concordância e fidedignidade de um questionário eletrônico para crianças (WEBDAFA). Rev Bras Cineantropom Desempenho Hum. 2013;15(1):38-48

24. Looij-Jansen PM, Wilde EJ. Comparison of Web-Based versus Paper-and-Pencil Self-Administered Questionnaire: Effects on Health Indicators in Dutch Adolescents. Health Serv Res. 2008:43(5):1708-21.

25. Mangunkusumo RT, Moorman PW, Ruiter AV, Lei J, Koning HJ, Raat H. Internet-administered adolescent health questionnaires compared with a paper version in a randomized study. J Adolesc Health. 2005;36:70e1-e6.

26. Romero A, Florindo AA, Voci SM, Slater B. Reprodutibilidade de questionário informatizado de atividade física em adolescentes. Rev Bras Ativ Fís Saúde. 2011;16(3):234-39.

27. Scheers T, Philippaerts R, Lefevre J. Assessment of physical activity and inactivity in multiple domains of daily life: a comparison between a computerized questionnaire and the SenseWear Armband complemented with an electronic diary. Int J Behav Nutr Phys Act. 2012;9(71):1-15.

28. Turner-Bowker DM, Saris-Baglama RN, DeRosa MA. Single-item electronic administration of the SF - 36 V2 Health Survey. Qual Life Res. 2012:1-6.

29. Omote S, Prado PT, Carrara K. Versão eletrônica de questionário e o controle de erros de resposta. Psicol Estud. 2005;10(3):397-405. 